\title{
Mobile Robot Local Path Planning Based on Improved T-S Fuzzy Neural Network
}

\author{
Suying Zhang, Yankai Shen, Zexu Sun \\ College of Electrical Engineering, Hebei University of Science and Technology, \\ Shi Jiazhuang, China \\ 1164492565@qq.com
}

\begin{abstract}
Keywords: T-S fuzzy neural network; local path planning; transformative Gaussian function; modified BP algorithm
\end{abstract}

\begin{abstract}
Aiming at the problems that BP algorithm has slow convergence rate and is likely to fall into local minimum point. This paper presents a modified fuzzy neural network which takes the distances between the robot and obstacles as input variables, and the velocity and the steering angle of the robot as output variables. Besides, the accurate environmental information can be obtained by fusing the information of ultrasonic sensors, and the network parameters can be adjusted by training the network. With the application of Matlab simulation software, the result shows that the improved fuzzy neural network has good performances in optimizing the convergence speed, learning speed and stability of the network.
\end{abstract}

\section{Introduction}

At present, the mobile robot path planning is one of the hot points in the field of intelligent robot research. The path planning is that how a robot searches out an optimal or sub-optimal path from the starting position to the target position according to some certain performance indexes [1]. Depending on how much the environmental information the robot acquires, the path planning strategies are divided into two kinds: one is global path planning, the other one is local path planning.

Up till now, some global path planning approaches, that the environment information has already known, have good performances. However, some means dealing with those situations where the environment is complex and time-varying are still insufficient. Especially the aspects of the local path planning and information acquisition, which make them become the future development direction of the path planning [2].

This article will combine the fuzzy logic algorithm with neural network making the network has the advantages of both being easy to express human knowledge and learning ability. What's more, an improved algorithm to solve the problem existing in traditional BP algorithm is proposed, and a optimal path is generated.

\section{The environmental information acquisition}

The distance information from the direction of left, right and front can be obtained by using ultrasonic sensors uniformly distributed around the robot (See Fig.1(a)). Assuming that the robot is viewed as a particle, the simplified diagram is shown in Fig.1(b). The angle between two adjacent sensors is $30^{\circ}$, and the effective measurement distance ranges from $2 \mathrm{~cm}$ to $100 \mathrm{~cm}$ [3].

Presuming that $d_{i}(i=1,2, \ldots, 6)$ is the ith sensor value, then the distance values fused are expressed as:

$$
D_{L}=\min \left(d_{4}, d_{5}, d_{6}\right), D_{F}=\min \left(d_{3}, d_{4}\right), D_{R}=\min \left(d_{1}, d_{2}, d_{3}\right) \text {. }
$$

Where $\mathrm{D}_{\mathrm{L}}$ means left-distance, $\mathrm{D}_{\mathrm{F}}$ means front-distance, $\mathrm{D}_{\mathrm{R}}$ means right-distance. 


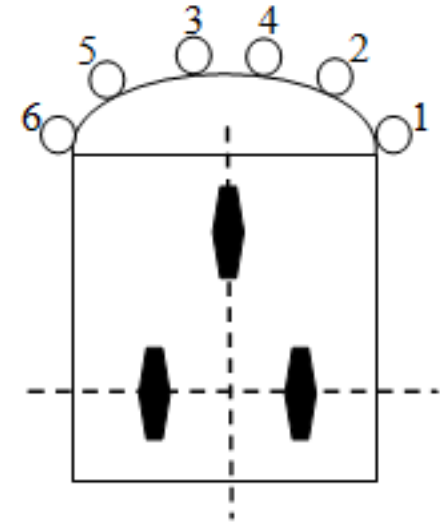

(a)

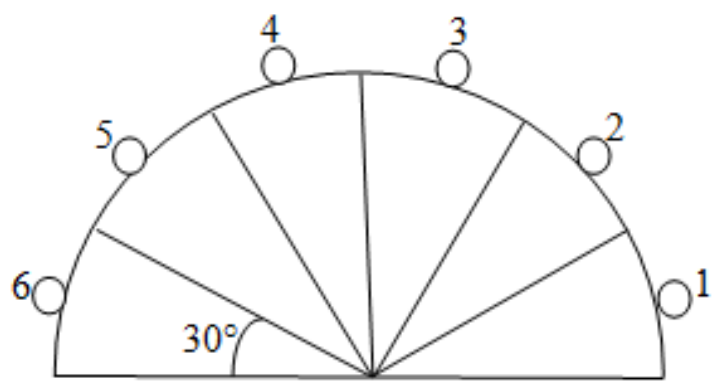

(b)

Fig.1 Ultrasonic Sensors Layout

\section{Design of fuzzy-neural network}

\subsection{Fuzzification}

The linguistic values of input variables are:

$$
\mathrm{D}=\{\mathrm{VN}, \mathrm{RN}, \mathrm{MD}, \mathrm{RF}, \mathrm{VF}\} \text {. }
$$

Where $\mathrm{D}$ includes $\mathrm{D}_{\mathrm{L}}, \mathrm{D}_{\mathrm{F}}$ and $\mathrm{D}_{\mathrm{R}}$; VN stands for very-near, $\mathrm{RN}$ for relatively-near, $\mathrm{MD}$ for moderate-distance, $\mathrm{RF}$ for relatively-near, $\mathrm{VF}$ for very-far.

The linguistic values of output variables are:

$\mathrm{V}=\{\mathrm{S}, \mathrm{M}, \mathrm{F}\}$

$\theta=\{\mathrm{TL}, \mathrm{TLL}, \mathrm{F}, \mathrm{TRL}, \mathrm{TR}\}$.

For the term set $\mathrm{V}, \mathrm{S}$ stands for slow, $\mathrm{M}$ for moderate, $\mathrm{F}$ for fast; For the term set $\theta$, TL stands for turn-left, TLL for turn-left-little, F for forward, TRL for turn-right-little, TR for turn-right.

\subsection{Structure of fuzzy-neural network}

Adaptive network-based fuzzy inference system is one of hybrid intelligent fuzzy-neural structure and it functions under T-S-type fuzzy interference system, which was designed by Jang in 1993 [4].The inference system if-then rules based on experts' experience and knowledge are defined as:

Rule: IF $x_{1}$ is $A_{i}$ and $x_{2}$ is $B_{i}$ and $x_{3}$ is $C_{i}$,

$$
\text { THEN } \mathrm{f}_{\mathrm{j}}=p_{j} \mathrm{x}_{1}+q_{j} \mathrm{x}_{2}+r_{j} \mathrm{x}_{3}+u_{j} \text {. }
$$

Where $A_{i}, B_{i}$ and $C_{i}$ are the fuzzy membership sets for the input variables $D_{L}\left(x_{1}\right)$, $\mathrm{D}_{\mathrm{R}}\left(\mathrm{x}_{2}\right), \mathrm{D}_{\mathrm{F}}\left(\mathrm{x}_{3}\right)$ respectively. $\mathrm{i}=1,2, \ldots, 5, \mathrm{j}=1,2, \ldots 125 ; p_{j}, q_{j}, r_{j}$ and $u_{j}$ are the linear parameters of function $f_{j}$ and changing these parameters we can modify the output of the controller [5].

Assuming that $Y_{i}$ is the output vector of the ith layer, $y_{i j}$ is the output between the ith neuron from last layer and the jth neuron of the next layer. Then, the function of each layer in fuzzy-neural network structure (See Fig.2) is discussed as follows:

Input layer: In this layer, nodes receive signals from array of sensors which specify the position of the obstacles.

First layer: This layer is the adaptive fuzzy layer. Neurons in this layer complete the 
fuzzification process. Every node in this stage is an adaptive node and calculating the membership function value in fuzzy set. For three inputs the outputs from nodes in this layer are presented as:

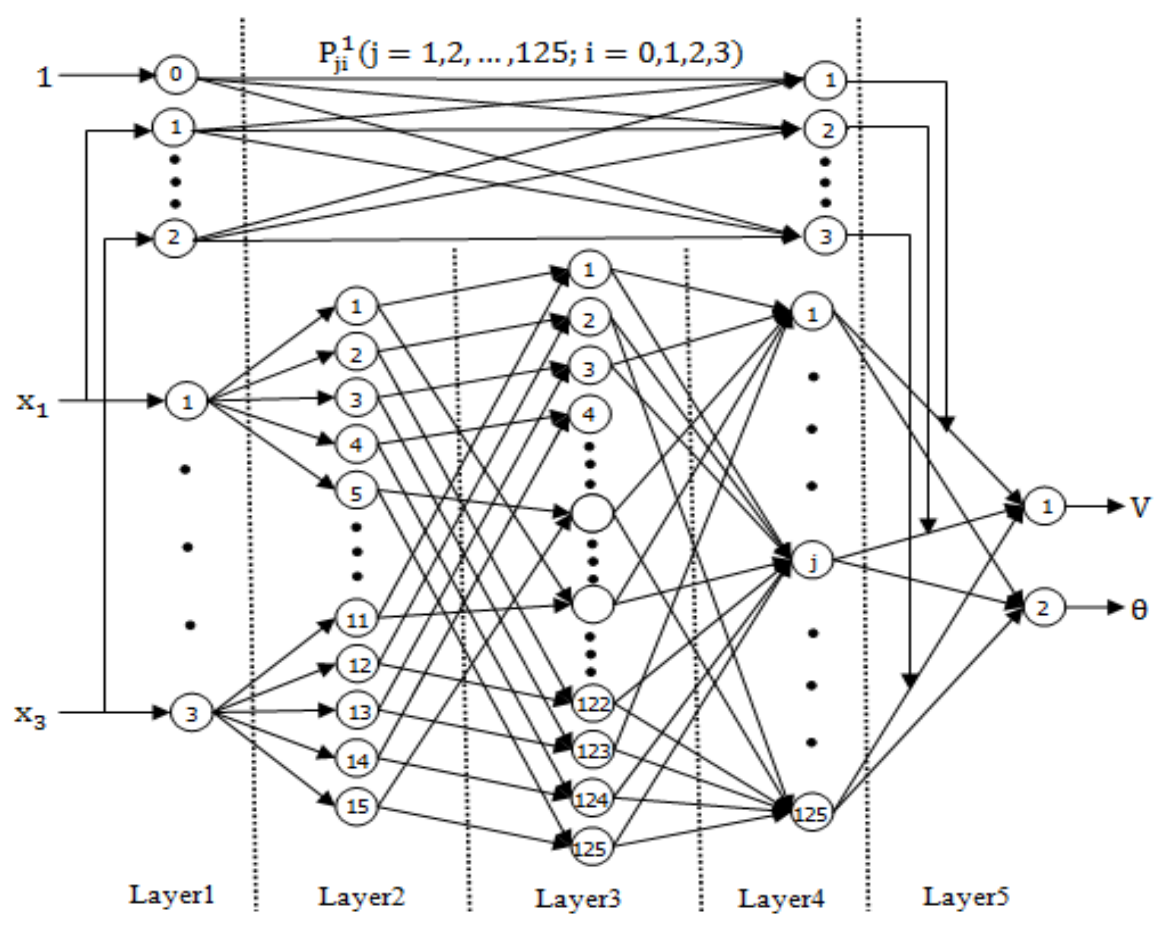

Fig.2 The Structure of Fuzzy-neural Network

$\mathrm{Y}_{2}=\left[\mu_{\mathrm{ij}}\right]_{15}^{\mathrm{T}}$.

The input membership functions are the transformative Gaussian function and defined as follows:

$\mu_{i j}=\exp \left(-b_{i j}\left(x_{i}-c_{i j}\right)^{2}\right), b_{i j}>0, b_{i j}=\frac{1}{2 \sigma_{i j}^{2}}$.

Where $\mathrm{c}_{\mathrm{ij}}, \sigma_{\mathrm{ij}}$ are parameters that control the centre, width respectively. Changing these parameters will give the various contour of the function as required in accordance with the data set for the problem defined. Not only does this novel form shorten the training time, but also accelerate the running speed of the network significantly [6].

Second Layer: It is known as the rule layer. Every node in this stage corresponds to a single T-S fuzzy rule. Each rule point receives inputs from the respective points of layer- 2 and calculates the firing strength of the each fuzzy rule. Output from each node is the product of all incoming signals.

$$
\begin{aligned}
& Y_{3}=\left[y_{3 j}\right]_{125 \times 1} \cdot \\
& y_{3 j}=\mu_{i_{1} 1} \cdot \mu_{i_{2} 2} \cdot \mu_{i_{3} 3} \cdot \mu_{i_{4} 4} \cdot \mu_{i_{5} 5} ; i_{1}, i_{2}, i_{3}, i_{4}, i_{5} \in\{1,2,3,4,5\}
\end{aligned}
$$

Third Layer: It is the normalization layer. Each point in this layer receives inputs from all points in the fuzzy rule layer and calculates the normalized firing strength of a given rule. The normalized firing strength of the jth point of the jth rules firing strength to sum of all rules firing strength.

$$
\begin{aligned}
& Y_{4}=\left[y_{4 j}\right]_{125 \times 1} \cdot \\
& y_{4 j}=\mu_{i_{1} 1} \cdot \mu_{i_{2} 2} \cdot \mu_{i_{3} 3} \cdot \mu_{i_{4} 4} \cdot \mu_{i_{5} 5} / \sum_{j=1}^{125} y_{3 j} .
\end{aligned}
$$

Output layer: Each node in this layer is connected to the corresponding normalization node and 
also receives initial inputs $\mathrm{x}_{1}, \mathrm{x}_{2}, \mathrm{x}_{3}$. A defuzzification node determines the weighted consequent value of a given rule presented as:

$$
\begin{aligned}
& \mathrm{Y}_{5}=\mathrm{Y} * \mathrm{Y}_{4}=[\mathrm{V}, \theta]^{\mathrm{T}} . \\
& \mathrm{Y}=\left[\mathrm{y}_{5 \mathrm{j}}\right]_{2 \times 125} . \\
& \mathrm{y}_{5 \mathrm{j}}=\sum_{\mathrm{i}=0}^{3} \mathrm{p}_{\mathrm{ji}} * \mathrm{x}_{\mathrm{i}}=\mathrm{p}_{\mathrm{j} 0} * 1+\mathrm{p}_{\mathrm{j} 1} * \mathrm{x}_{1}+\mathrm{p}_{\mathrm{j} 2} * \mathrm{x}_{2}+\mathrm{p}_{\mathrm{j} 3} * \mathrm{x}_{3} .
\end{aligned}
$$

\section{Modified learning method}

The cost function and the parameter adjust algorithm are defined as:

$$
\begin{aligned}
& E=\frac{1}{2} \sum_{k=1}^{5}\left(O_{k}-Y_{k}\right)^{2} . \\
& p_{j i}(k+1)=p_{j i}(k)-\eta \frac{\partial E}{\partial p_{i j}}(k) . \\
& c_{i j}(k+1)=c_{i j}(k)-\eta \frac{\partial E}{\partial c_{i j}}(k) . \\
& \sigma_{i j}(k+1)=\sigma_{i j}(k)-\eta \frac{\partial E}{\sigma_{i j}}(k) .
\end{aligned}
$$

Where $\mathrm{O}_{\mathrm{k}}$ represents the desired output, $\mathrm{Y}_{\mathrm{k}}$ is on the behalf of the actual output, and $\eta(0<\eta<$ 1 ) is on the behalf of the learning efficiency.

In order to solve the local minimum problem existing in the traditional BP algorithm, a correction method based on Romberg is proposed, taking the $\mathrm{p}_{\mathrm{ji}}$ for example.

$$
\begin{aligned}
& \mathrm{S}^{\mathrm{k}-2}=\frac{4 \mathrm{P}^{\mathrm{k}-2}-\mathrm{P}^{\mathrm{k}-3}}{3}, \mathrm{~S}^{\mathrm{k}-1}=\frac{4 \mathrm{P}^{\mathrm{k}-1}-\mathrm{P}^{\mathrm{k}-2}}{3}, \mathrm{~S}^{\mathrm{k}}=\frac{4 \mathrm{P}^{\mathrm{k}}-\mathrm{P}^{\mathrm{k}-1}}{3} . \\
& \mathrm{C}^{\mathrm{k}-1}=\frac{16 \mathrm{~S}^{\mathrm{k}-1}-\mathrm{S}^{\mathrm{k}-2}}{15}, \mathrm{C}^{\mathrm{k}}=\frac{16 \mathrm{~S}^{\mathrm{k}}-\mathrm{S}^{\mathrm{k}-1}}{15} . \\
& \mathrm{P}^{\mathrm{k}}=\frac{64 \mathrm{C}^{\mathrm{k}}-\mathrm{C}^{\mathrm{k}-1}}{63} .
\end{aligned}
$$

Where $\mathrm{P}^{\mathrm{k}-3}, \mathrm{P}^{\mathrm{k}-2}, \mathrm{P}^{\mathrm{k}-1}, \mathrm{P}^{\mathrm{k}}$ represent the training vectors of every four times. $\varepsilon$ is an arbitrarily small positive number. If $\mathrm{E}>\varepsilon$, then make $\mathrm{E}=0$ and judge whether $\mathrm{P}^{\mathrm{k}-3}, \mathrm{P}^{\mathrm{k}-2}, \mathrm{P}^{\mathrm{k}-1}, \mathrm{P}^{\mathrm{k}}$ have saved? If so, use the Romberg method amend the vector values, and keep the correction value $\mathrm{P}^{\mathrm{k}}$ as the next training data. Otherwise, stop training, and output the training results.

\section{Simulation}

This part mainly describes the simulation results of the improved T-S-type neural network. The initial membership functions is shown in Fig.4. After adjusting the parameters of the membership function, the centre and width positions of the membership functions become more fit to the actual control requirement (See Fig.5). From Fig.6, we can see, when the robot is in the situation where obstacles are concentrated, it has the possibility to hit obstacles. After using the modified learning 
method to amend the training vectors, the robot can bypass all obstacles, and the generated path is much more smooth then before as it is shown in Fig.7.

\section{Conclusion}

This article puts forward two improved points based on T-S-type fuzzy neural network, namely: the membership function is the deformation form of Gaussian function and the learned parameters are corrected by the Romberg method. This solution not only improves the convergence speed and learning efficiency, but also effectively solves the local minima problem existing in BP algorithm, and the simulation results also verify the feasibility and effectiveness of the proposed solution.

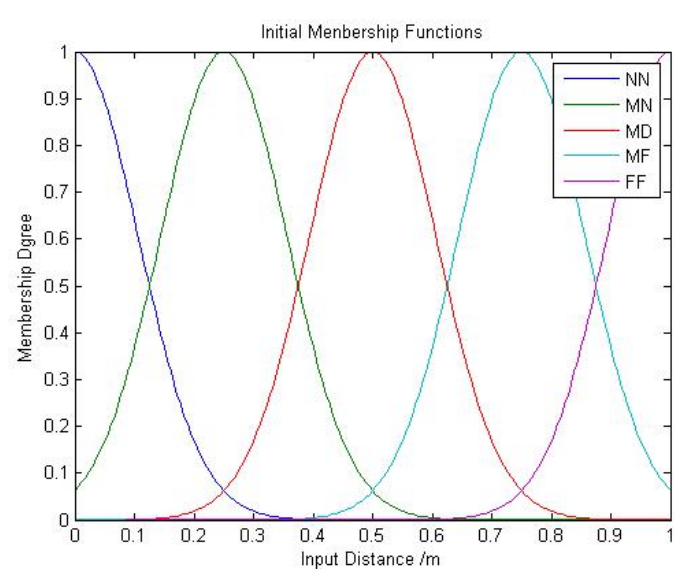

Fig.4 The Initial Membership Functions

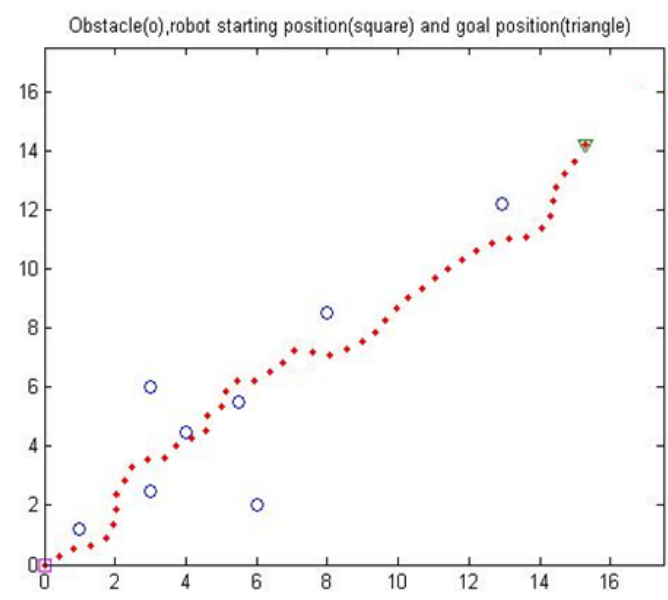

Fig.6 The Path Generated with The Traditional Method

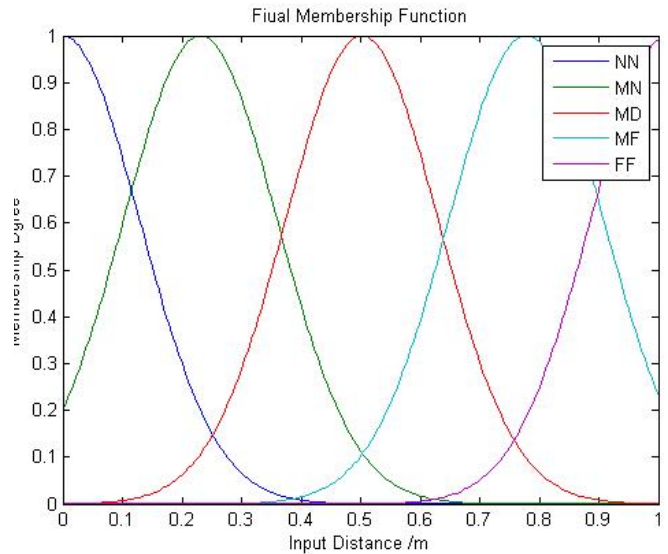

Fig.5 The Final Membership Functions

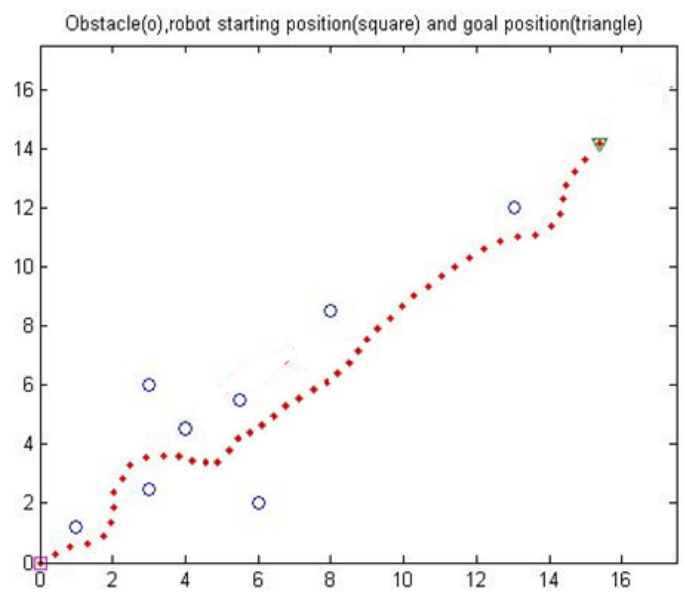

Fig.7 The Path Generated with The Modified Method

\section{References}

[1] Liang Jin, Song Ke-pu: Journal of System Simulation Vol.269-272(2010).

[2] Zhang Han-dong, Zhang Rui, Cen Yu-wan: Journal of System Simulation Vol.439-443(2005).

[3] Xiong Kai-feng, Zhang Hua: Manufacturing Automation Vol.1-9(2013).

[4] J.S.R. Jang: Adaptive network-based fuzzy inference system. IEEE Transaction on System, Man and Cybernetics -part B Vol. 665-685 (1993).

[5] Prases K. Mohanty, Dayal R. Parhi: Applied Mathematics \& Information Sciences Vol. 25272535(2014).

[6] Wei Wu, Long Li, Jie Yang, Yan Liu: Information Sciences Vol. 1630-1642(2010). 